CU-TP-878

\title{
Baryon Junction Stopping at the SPS and RHIC via HIJING/B
}

\author{
S.E. Vance ${ }^{a}$ 円, M.Gyulassy ${ }^{a}$ X.N. Wang $^{b}$ \\ ${ }^{a}$ Physics Department, Columbia University, New York, NY, 10027 \\ ${ }^{b}$ Nuclear Science Division, LBNL, Berkeley, CA 94720
}

Baryon stopping at the SPS and RHIC energies is calculated by introducing a new baryon junction mechanism into HIJING. The exchange of a baryon junction, according to Regge phenomenology, leads to a $\cosh \left[\left(y-y_{C M}\right) / 2\right]$ rapidity dependence and an $1 / \sqrt[4]{s}$ energy dependence of the inclusive baryon cross section. This baryon junction dynamics also leads naturally to enhanced $p_{T}$ broadening in $p A$ and $A A$ together with enhanced mid-rapidity hyperon production.

\section{Introduction}

The systematic study of baryon stopping and hyperon production in $p p, p A$, and $A A$ collisions is essential in order to differentiate the sought after new physics of dense equilibrated matter from non-equilibrium multi-particle production dynamics[1]. Baryon stopping refers to the transport of baryon number in rapidity space away from the nuclear fragmentation regions and is measured through the single inclusive rapidity distribution of protons and hyperons. As discussed in [2,3], recent SPS data shows a high degree of baryon stopping and anomalous hyperon production. These findings are based on comparisons of predictions using the HIJING [5] and VENUS [6] Monte Carlo event generators with SPS data for $p+A$ and $A+B$ collisions. In particular, the baryon stopping mechanism modeled in HIJING was found to underestimate the stopping in heavy nuclear collisions, while VENUS model was able to reproduce the baryon stopping and strange baryon production by introducing a new non-equilibrium dynamical mechanism called the double string. The mechanism in HIJING, adapted from the LUND Fritiof [7] and dual parton model (DPM) [8], involves diquark-quark string excitations followed by string breaking. The final valence baryons in this model always emerge within about a unit of rapidity from those of the unbroken diquarks. This association of the baryon with the end point diquark creates a characteristic baryon rapidity distribution where the baryons are concentrated near the beam and target fragmentation regions as shown in Fig 1a. The extrapolation of the $p p$ physics of HIJING to $P b P b$ is seen to underestimate the mid-rapidity valence baryon yield by a factor of two in Fig 1b. VENUS avoids this "fly trap" by postulating a specific model of diquark breakup, leading to double strings. The double string emerging from a broken diquark is assumed to loop around a valence quark from the other fragmentation region. The produced baryon is then associated with the fragmentation of the double string near its $180^{\circ}$ bend. The parameters of this mechanism are adjusted from $p A$ and suffice to reproduce the $A A$ data. We emphasize that the

\footnotetext{
${ }^{1}$ Presented at QM97, Tsukuba, Japan
} 
analytic one parameter Multi-Chain-Model [4] also reproduces well[3] the valence proton distributions based on fitting $p A$. The advantage of a Monte Carlo event generator is the ability to test via other observables such as hyperon production and $p_{\perp}$ systematics the consistency of specific dynamical assumptions with a wide range of data.

Another model of baryon transport was proposed by Kopeliovich and Zakharov [9] based upon pQCD considerations. That mechanism has been recently included in a new version of the DPM [10]. In that model, the leading diquark is broken by a color exchange which changes its color state from a $\{\overline{3}\}$ to a $\{6\}$. This allows one of the valence quarks to flow into the central rapidity region and act as the seed for the production of the final baryon number. The inclusive cross section of this mechanism is characterized by a cosh $y / 2$ rapidity dependence and an $1 / \sqrt[4]{s}$ energy dependence which follows from the assumed $1 / \sqrt{x}$ distribution of the valence quarks in a proton. In practice this mechanism is similar to that invoked in VENUS in that the fragmentation of the now two independent quarks from the diquark is similar to that from two independent strings. As in VENUS this configuration allows for a factor of 2 enhancement in the production of strange baryons. Adding a component of about $20 \%$ for each nucleon-nucleon collision, with the assumption that once the diquark is broken then it remains broken, DPM is then also able to reproduce the SPS data.

In this work we test a third model of baryon transport recently proposed by Kharzeev [11] and based on a long dormant Rossi-Veneziano baryon junction Regge exchange model[12]. In this approach, the baryon number is traced by a non-perturbative baryon junction of topological nature. The special theoretical appeal of that idea arises from the form of the baryon wavefunction in QCD. The requirement of gauge invariance of the nonlocal operator creating a baryon naturally leads to the concept of a baryon junction:

$$
\begin{aligned}
B\left(x_{1}, x_{2}, x_{3}, x_{J}\right)=\epsilon^{i j k}\left[P \exp \left(i g \int_{x_{1}}^{x_{J}} d x^{\mu} A_{\mu}\right) q\left(x_{1}\right)\right]_{i} & {\left[P \exp \left(i g \int_{x_{2}}^{x_{J}} d x^{\mu} A_{\mu}\right) q\left(x_{2}\right)\right]_{j} } \\
& \times\left[P \exp \left(i g \int_{x_{3}}^{x_{J}} d x^{\mu} A_{\mu}\right) q\left(x_{3}\right)\right]_{k} .
\end{aligned}
$$

The baryon junction is a vertex at $x_{J}$ where the three gluon Wilson lines emanating from the three valence quarks (in $\mathrm{SU}(3)$ ) must join in order to form a gauge invariant operator. In a highly excited baryonic state, the three valence quarks fragment via multiple $q \bar{q}$ into mesons leaving three sea quarks eventually around the junction to form the observed final baryon. This is the sense in which the junction traces the baryon number. Being a purely gluonic configuration, the junction may be easily transported into the mid-rapidity region. The fragmentation of the end point valence quarks naturally leads to three beam jets, very similar to the previous mechanisms considered. The primary advantage of this mechanism is that Regge phenomenology can be used to estimate the inclusive inelastic cross section for this process. From $p \bar{p}$ and $p p$ data, the junction-anti-junction exchange is characterized by a trajectory with intercept, $\alpha_{J \bar{J}}(0) \approx 1 / 2$. This leads immediately to a $\cosh y / 2$ rapidity dependence and an $1 / \sqrt[4]{s}$ energy dependence.

The unique prediction of this mechanism is that fragmentation of the valence quarks down to the junction is expected to enhance hyperon production by a factor of 3 just from the random combinatorics of $s$ vs $u, d$ pair production. In addition, from the random addition of three sea quarks, the transverse momentum of the final baryon is automatically 

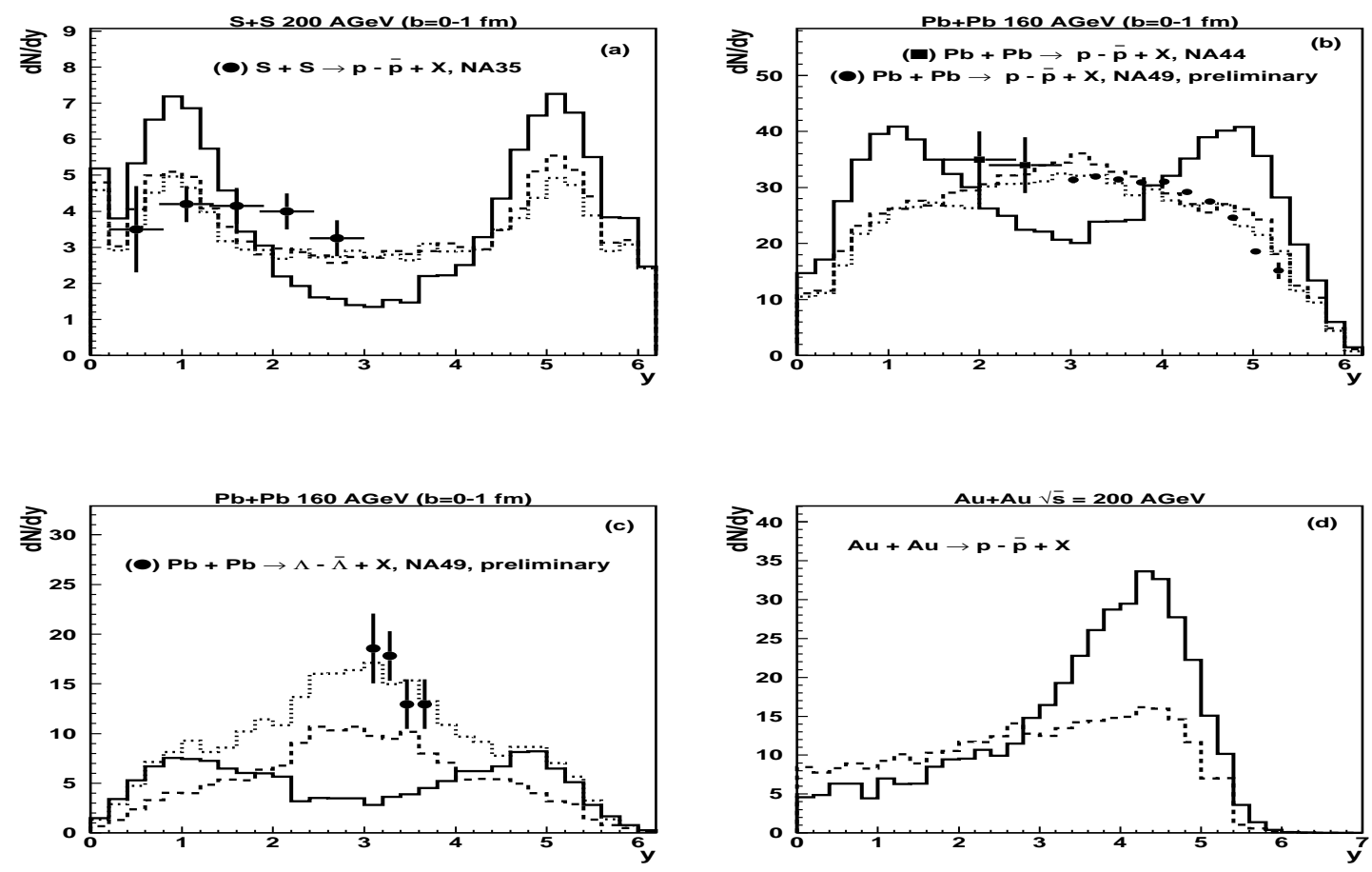

Figure 1. A comparison of HIJING (solid), HIJING/B (dashed) and HIJING/B with "ropes" (dotted) with various experimental data.

enhanced by a factor of $\sqrt{3}$.

Given these interesting coupled consequences of the baryon exchange mechanism, we have chosen to implement this model in a new version of HIJING, called HIJING/B 13.

\section{HIJING/B}

In HIJING/B, baryon junction stopping is implemented using a "Y" string configuration for the excited baryons in which the baryon junction and produced baryon are distributed as noted above. The resulting three beam jets are treated as $q \bar{q}$ configurations. For each nucleon-nucleon interaction, a $\sim 60 \%$ probability is given that only one of the two nucleons will have its baryon junction stopped in the central rapidity region. Baryon excitations without junction exchange are taken to be standard $(q q)-q$ strings for fragmentation purposes. Due to kinematical considerations, junction exchange is allowed only if the invariant mass exceeds $m \geq 4.5 \mathrm{GeV}$ so that the three beam jets have room to decay. At SPS energies this trivial kinematic constraint limits considerably the number of junction exchanges allowed. The junction exchange probability is fitted so as to reproduce the $p p$ valence proton data. For multiple collisions, we assume that once the baryon junction is exchanged, it remains stopped in subsequent soft interactions.

A comparison of the prediction of HIJING and HIJING/B with SPS data 14 16] is given in Fig 1a,b,c. In part (a), (b) we show the valence proton $(p-\bar{p})$ rapidity distribution for, $S+S$ and $P b+P b$, respectively. Whereas HIJING under-predicts the baryon stopping, 
HIJING/B is shown to provide a large amount of stopping. The valence hyperon $(\Lambda-\bar{\Lambda})$ rapidity distribution is shown in part (c) of Fig. 1. Here, HIJING/B provides a factor of 3 more valence $\Lambda$ at mid-rapidity consistent with the expected strangeness enhancement associated with the baryon junction exchange. However the very large value of the valence hyperon yield in $\mathrm{PbPb}$ cannot be reproduced. Multiple final state interactions or "rope" effects must be included to understand those yields. Increasing the effective string tension by a factor of two makes little difference on the valence non-strange baryon yields, but can account for the anomalous hyperon yield (dotted curves).

Finally in Fig 1d, the predictions of this model for the valence proton rapidity distribution in $A u+A u$ collisions at RHIC energies $(\sqrt{s}=200 \mathrm{GeV})$ are shown. HIJING/B predicts nearly twice the initial baryon density, $\rho_{B}(1 \mathrm{fm}) \approx 2 \rho_{0}$, at mid-rapidity than HIJING.

In conclusion, a novel baryon junction stopping mechanism is implemented in HIJING/B to reproduce baryon transport in $p p$ collisions. This is sufficient to account for the midrapidity valence baryon data in $p A$ and $A A$. The model naturally leads to large enhancement of the $p_{\perp}$ slope as well as of the yield of hyperons. However, the preliminary $\Lambda-\bar{\Lambda}$ yield in $\mathrm{PbPb}$ is still underestimated without multiple final state interactions.

Acknowledgements: We thank Dima Kharzeev for stimulating discussions. This work was supported in part by the Director, Office of Energy Research, Division of Nuclear Physics of the Office of High Energy and Nuclear Physics of the U.S. Department of Energy under Contract No. DE-FG02-93ER40764.

\section{REFERENCES}

1. M. Gyulassy, Nucl. Phys. A590 (1995) 197c.

2. V. Topor Pop et al., Phys. Rev. C52 (1995) 1618.

3. M. Gyulassy, V. Topor Pop and S.E. Vance, Heavy Ion Physics 5 (1997) 299.

4. S. Date, M. Gyulassy and H. Sumiyoshi, Phys. Rev. D32, 619 (1985).

5. X. N. Wang and M. Gyulassy, Phys. Rev. D44 (1991) 3501; Phys. Rev. D45 (1992) 844; Comp. Phys. Comm. 83 (1994) 307.

6. K. Werner, Phys. Rep. 232 (1993) 87.

7. B. Andersson et al., Nucl. Phys. B281 (1987) 289; Comp.Phys.Comm. 43(87)387.

8. A. Capella et al., Phys. Rep. 236 (1994) 225.

9. B.Z. Kopeliovich and B.G. Zakharov, Z. Phys. C, 43, (1989) 241.

10. A. Capella, B.Z. Kopeliovich, Phys. Lett. B381 (1996) 325, hep-ph/9603279;

11. D. Kharzeev, Phys. Lett. B378 (1996) 238. nucl-th/9602027.

12. G.C. Rossi and G. Veneziano, Nucl.Phys. B123 (1977) 507; Phys. Rep. 63 (1980) 153.

13. S.E. Vance, M. Gyulassy and X.N. Wang, to be published.

14. T. Alber et al., (NA35 Collaboration), submitted to Z. Phys. C; J. Bächler et al., (NA35 Collaboration), Phys. Rev. Lett 72 (1994) 1419.

15. N. Xu et al. (NA44 Collaboration), Nucl. Phys. A610 (1996) 175c; J. Bearden et al., (NA44 Collaboration), Phys. Lett. B372 (1996) 339.

16. P. Jacobs, (NA49 Collaboration), Proc. ICPAQGP97, Jaipur, India (1997); C. Bormann, (NA49 Collaboration), Proc. of Strangeness in Quark Matter 97. 